\title{
Enfoque espacial y visiones territoriales del desarrollo regional en el Ecuador (1952-1982). Estudios, programas y planes
}

\author{
Anna Vincenza Nufrio
}

Afiliación: Universidad de Cuenca, Cuenca, Ecuador.

\section{Email: anna.nufrio@ucuenca.edu.ec}

Recibido: 2 de junio del 2017

Aceptado: 13 de noviembre del 2017

\section{Anna Vincenza Nufrio}

Arquitecta y Doctora por el Politecnico di Milano. Docente en Universidades europeas (ESARQ/ UIC, UPC, UPM, POLIMI) y latinoamericanas (UJTD, UNAL, CUJAE, UCUENCA). Investigadora principal de programas de concurrencia competitiva internacional: Rientro dei Cervelli (MIUR, Italia); Marie Curie (UE-UPM, España); Prometeo (SENESCYT, Ecuador). Miembro del Grupo de Investigación en Economía Regional (GIER), Facultad de Ciencias Económicas y Administrativas, Universidad de Cuenca, Ecuador. Investiga sobre: Planificación Urbana Transfronteriza; Planificación Urbana, Regional y Micro-regional; Historia del Hábitat y Producción Social del Hábitat.

\begin{abstract}
Resumen
Los cambios de escala de los procesos políticos-económicos impuestos por la globalización orientan cada vez más, en Europa, Asia y América Latina, las prácticas de la planificación territorial hacia la implementación de estrategias de desarrollo regional y sub-regional. La "regionalización" es un tema pendiente en la agenda política del Ecuador, y no obstante la actual Constitución establece que el país se organiza territorialmente en regiones, provincias, cantones y parroquias rurales, el desarrollo equitativo, la autonomía y la descentralización de los distintos niveles de gobierno siguen siendo objetivos a alcanzar. Este artículo respalda este debate indagando sobre el enfoque espacial y las visiones territoriales que entre 1952 y 1982 orientaron las propuestas de regionalización en el Ecuador. A tal propósito se analizan valiosos documentos inéditos (estudios, planes y proyectos) realizados por la JUNAPLA y el CREA: únicos ensayos de Planificación Regional realizados en el país, de manera particular en la Región Centro-Sur.
\end{abstract}

Palabras Clave: CREA; desarrollo regional; JUNAPLA; planificación física regional.

Spatial approach and territorial views of the regional development in Ecuador (1952 - 1982). Studies, projects and plans

\begin{abstract}
The change of scale of the political-economic processes imposed by globalization are increasingly orienting, in Europe, Asia and Latin America, the territorial planning practises towards the implementation of regional and sub-regional development strategies. "Regionalization" is a pending issue in Ecuador's political agenda and, although the current Constitution establishes that the country is organized territorially in regions, provinces, cantons and rural parishes, equitable development, autonomy and decentralization of the different government levels remain an objective to achieve. This article supports this debate by investigating the spatial approach and the territorial views that guided the regionalization proposals in Ecuador between 1952 and 1982. To this end, valuable documents (studies, plans and projects) carried out by the JUNAPLA and the CREA have been analyzed: unique attempts of Regional Planning carried out in the country, especially in the Center-South Region.
\end{abstract}

Keywords: CREA; JUNAPLA; regional development; regional spatial planning. 


\section{Introducción}

$\mathrm{D}$

esde el año 2000, en muchos países de América Latina y el Caribe, se asiste a un creciente interés por incorporar el enfoque territorial regional en diversos ámbitos de la acción del Estado: reformas constitucionales, construcción de visiones territoriales de largo plazo, impulso a procesos de descentralización y nuevas políticas nacionales de desarrollo territorial, entre otros. La "cuestión regional" retoma protagonismo en las agendas gubernamentales, principalmente en el contexto de los debates relativos a la reforma democrática del Estado basada en la descentralización y las autonomías (Valarezo, G. R., Báez Rivera, S. y Ospina Peralta, P., 2004). Principalmente en algunos países andinos como Bolivia, Ecuador y Venezuela, el giro hacia la izquierda de sus Gobiernos y la aparición de poderosos movimientos de reivindicación étnica y social abren espacios para nuevos debates sobre el fomento de políticas de desarrollo regional (Moncayo, 2013).

La regionalización del Ecuador se halla en un completo estado de abandono, no sólo porque la planificación nacional y regional ha estado ausente de la gestión pública en el pasado reciente, sino también porque en la definición territorial incluida en la nueva Constitución, por un lado se habla de regiones y por el otro se crean gobiernos autónomos descentralizados (Pauta, 2013). La Constitución del Ecuador (Asamblea Nacional Constituyente, 2008), en su Capítulo II, relativo a la "Organización del Territorio", y de acuerdo al Código Orgánico de Organización Territorial, Autonomías y Descentralización (Asamblea Nacional Constituyente, 2010) establece que el territorio nacional debe organizarse en regiones, provincias, cantones y parroquias rurales $y$, en su Primera Disposición Transitoria, numeral 9, fija como límite temporal para la conformación de regiones un plazo de ocho años. Sin embargo, la Asamblea Nacional, mediante la aprobación de la Enmienda consignada en el Artículo 15, RO. No. 653 Suplemento del lunes 21 de diciembre de 2015, ha derogado dicha obligatoriedad en los plazos anteriormente establecidos, eliminando la limitación temporal y disponiendo que la Ley que regule la descentralización territorial de los distintos niveles de gobierno y el sistema de competencias fije el plazo para su conformación.

Ante la necesidad de reconstruir una institucionalidad democrática, también a escala territorial, para asumir retos que la actualidad requiere, es incomprensible cómo los actuales debates políticos y académicos sobre la planificación regional en el Ecuador son blandos, disciplinalmente desconectados, cuando no del todo inexistentes. Un silencio inoportuno que ocurre en un momento histórico en el cual la importancia creciente de las regiones en la reconfiguración del orden mundial está produciendo una

$1 \quad$ La investigación "Los desafíos de la planificación regional en Ecuador. Planeamiento estratégico sub-regional como herramienta eficaz para la reducción de asimetrías y desequilibrios territoriales, instrumento vertebrador de procesos de cooperación y coordinación territorial regional", ha sido dirigida por la Dra. Nufrio en el marco del PROGRAMA PROMETEO (SENESCYT, Ecuador) entre febrero 2015 y julio de 2016. Se agradece de manera especial al Ph.D Rodrigo Mendieta Muñoz y al M.Sc. Santiago E. Pozo Rodríguez, del Grupo de Investigación GIER, por la colaboración prestada en la revisión y orientación crítica de algunas partes del presente artículo.

2 La Región Centro-Sur de Ecuador, hoy "Zona 6" de planificación, incluía a las provincias de Azuay, Cañar y Morona Santiago.

3 El Archivo Histórico del ex CREA reposa en la Secretaría Nacional de Planificación y Desarrollo SENPLADES Zona 6-Austro, ubicado en la ciudad de Cuenca, Ecuador.

$4 \quad$ Los documentos editados por la JUNAPLA están archivados en los repositorios de las siguientes instituciones: Instituto de Altos Estudios Nacionales (IAEN); Universidades ecuatorianas; ILPES; CEPAL, entre otros. literatura especializada y una enorme variedad de conceptos y debates.

El presente artículo parte de una investigación más amplia ${ }^{1}$ y quiere ser una contribución a los estudios regionales en tema de planificación física y en el contexto latinoamericano. Su objetivo principal es revelar cuáles fueron los términos principales del debate sobre la planificación nacional y regional del desarrollo en el Ecuador entre 1952 y 1982: como este fue promovido por la Junta Nacional de Planificación y Coordinación Económica (JUNAPLA) a través de los Planes Nacionales de Desarrollo y en coordinación con el Centro de Reconversión Económica del Azuay, Cañar y Morona Santiago (CREA), en el contexto urbano-rural de la Región Centro-Sur y a través de un programa de envergadura regional. ${ }^{2}$

La investigación, que se sustenta en un enfoque metodológico histórico-crítico e interdisciplinario, basado en la consulta de valiosos documentos del Archivo Histórico del CREA ${ }^{3}$ y del Fondo Documental Digital de la JUNAPLA ${ }^{4}$, ha sido complementada por la edición/re-edición de mapas analíticos e interpretativos, útiles para la comprensión de los distintos planteamientos espaciales de regionalización propuestos durante 25 años de actividad planificadora.

El artículo se estructura en torno a cuatro argumentos principales: la institucionalización de la planificación nacional y regional en Ecuador; la introducción de los enfoques "espacial" y "regional" en los Planes Nacionales de Desarrollo; el CREA y el fomento de la planificación regional en la Región Centro-Sur; la formulación del Primer Plan Integral de Desarrollo Regional y la aplicación de las estrategias de Desarrollo Rural Integral (DRI). 
$5 \quad$ Hasta principios de los años $30 \mathrm{el}$ crecimiento económico en Latinoamérica se basó principalmente en la expansión de las exportaciones y en el aprovechamiento de las ventajas que ofrecían determinados recursos naturales, de interés para el mercado internacional.

6 Son conocidos los ensayos de planificación parcial realizados en México (desarrollo de la energía, transportes y riego); en Chile (planes de acción para el aprovechamiento hidroeléctrico, desarrollo de la siderurgia, exploración y explotación de petróleo); en Argentina (planes viales y de transportes) y en Brasil (fomento de la Industria siderúrgica, la energía y las carreteras)

$7 \quad$ "Región" significa en este contexto: los países de América Latina y el Caribe.

\section{$8 \quad$ La CEPAL fue establecida por la} resolución $106(\mathrm{VI})$ del Consejo Económico y Social, el 25 de febrero de 1948; es una de las cinco comisiones regionales de la ONU, con sede en Santiago de Chile.

$9 \quad$ ILPES, organismo que forma parte de la CEPAL. Fue creado en 1962 y tuvo la finalidad de apoyar a los Gobiernos de la Región en tema de planificación y gestión pública, mediante la prestación de servicios de capacitación, asesoría e investigación.

10 Notables son los estudios relativos a las condiciones y perspectivas para el crecimiento de los distintos países de América Latina.

\section{Entidad adscrita al Ministerio de} Economía, fue un Organismo de consulta, y no de ejecución. Sus funciones básicas fueron asesorar al Poder Público en materia de Planificación para el Desarrollo Económico-Social (nacional y regional) y coordinar la política económica de los organismos estatales en tema de inversiones nacionales o en el extranjero.

\section{Los principales programas sectoriales} fueron los de Energía y Transporte.

13 Comúnmente conocido como "Sombrero Panamá".

\section{La estructura productiva ecuatoriana} se basaba en la exportación de productos como el arroz, el café, el cacao y los productos de paja toquilla.

\section{Este proceso fue denominado "de} industrialización substitutiva": la industrialización se orienta, en principio, hacia la substitución con producción nacional de las importaciones de bienes de consumo no duraderos y duraderos y, en un segundo momento, se incorporan bienes intermedios y algunos bienes simples de la actividad metalmecánica. Esta estrategia de desarrollo fue sustentada por el capital extranjero, fundamentalmente a través de préstamos.

\section{La institucionalización de la planificación nacional en el Ecuador y la aplicación de los enfoques "físico" y "regional" en las políticas de desarrollo}

El interés por la planificación nacional del desarrollo surge en Latinoamérica durante la segunda postguerra cuando, al igual que en otros países desarrollados, el Estado asume por primera vez la conducción del proceso de desarrollo para transformar la estructura productiva ${ }^{6}$ mediante estrategias de industrialización y la planificación de la economía (Moncayo, 2013). Durante los primeros años de la década de 1950, la planificación comienza a institucionalizarse en diversos países de la región ${ }^{7}$ gracias a la orientación teórica, ideológica y técnica de la Comisión Económica para América Latina y el Caribe (CEPAL) ${ }^{8}$, para consolidarse en 1962 gracias a la creación del Instituto Latinoamericano de Planificación Económica y Social (ILPES, 1962). ${ }^{9}$ Específicamente, la CEPAL asesoró a los distintos Gobiernos en el tema de creación de Organismos Nacionales de Planificación (ONP) y en la formulación de Planes Nacionales de Desarrollo (PND); promovió estudios e investigaciones ${ }^{10} \mathrm{y}$ formó a programadores, funcionarios gubernamentales y especialistas en materias económicas (Martner y Máttar, 2012).

En el caso de Ecuador, si bien en los años 30 se producen los primeros intentos de asignar al Estado un rol organizador del desarrollo nacional (Peñaherrera Padilla, 1986), es con la Constitución del Ecuador de 1945 (Asamblea Nacional Constituyente, 1945) que se concibe la necesidad urgente de implementar la planificación económica para mejorar las condiciones de vida de la sociedad ecuatoriana. Ecuador fue el primer país latinoamericano que creó el 29 de mayo de 1954, mediante Decreto Ley de Emergencia Núm. 19, RO. Núm. 527, un Organismo Nacional de Planificación (ONP): la Junta Nacional de Planificación y Coordinación Económica (JUNAPLA). ${ }^{11}$

La planificación nacional en Ecuador se institucionalizó en dos fases: la primera, de iniciación y afianzamiento (1954-1960), y durante la cual se formularon planes sectoriales $^{12}$, se prepararon proyectos y estudios y se elaboraron presupuestos de capital; la segunda, de madurez y consolidación (1961-1965), y durante la cual se completaron varios estudios y se inició un periodo de formulación de PND. La aplicación de políticas de desarrollo económico tuvo un primer espacio colectivo a escala regional en las provincias de Azuay, Cañar y Morona Santiago (Regalado, 2011), a causa de las coyunturas económicas de principio de los 50: una grave crisis económica determinada por la dependencia de dichas provincias de la exportación del "sombrero de paja toquilla"13. Frente a esta urgencia, no pudiendo el Gobierno ecuatoriano aplicar el modelo de industrialización y de sustitución de las importaciones, en fase de implementación en otros países de América Latina, debido a la tradicional estructura productiva del país ${ }^{14}$, creó en ayuda de las provincias Azuayas mediante Decreto Ley de Emergencia de abril de 1952 el Instituto de Recuperación Económica del Azuay y Cañar (IRE): entidad que tenía la finalidad de reactivar la economía de la región, fortalecer las actividades agropecuarias y promover el fomento de las artesanías, de la pequeña industria y de la industria.

Los primeros años de actividad de la JUNAPLA (1951-1954) estuvieron caracterizados por una elevada estabilidad política, fundamentada en una alta prosperidad económica ${ }^{15}$, situación que facilitó la promoción de estudios sobre aquellas provincias, regiones 0 sectores económicos del país que requerían atención y ayudas adicionales urgentes. Se estudiaron, sobre todo, aspectos parciales de la economía de la provincia de Azuay y, en 1955, ya se habían elaborado los primeros Programas de Asistencia Técnica para las zonas deprimidas y emitido Decretos/Leyes de Emergencia para el fomento de Programas de Reconversión Económica (Galo, 1979).

La Sección de Estudios Generales del Departamento Técnico de la JUNAPLA finalizó en 1956 un primer estudio titulado "Azuay y Cañar: Desarrollo Económico. Situación Agraria y Forestal" (JUNAPLA, 1956), un análisis que revelaba cómo la economía de la Región Centro-Sur sufría de una depresión crónica, imputable a "una combinación de factores de orden natural, técnico agrícola y estructural" (JUNAPLA, 1956, $p$. 69). El IRE tuvo una existencia muy corta, debido a la promoción de actividades de 
carácter sectorial, implementadas de manera discontinua, y a la carencia de recursos económicos; razones que llevaron a que el 7 de noviembre de 1958 una nueva Ley, publicada en Registro Oficial $N^{0} 698$ de diciembre del mismo año, creara un nuevo Organismo de Desarrollo Regional: el Centro de Reconversión Económica del Azuay, Cañar y Morona Santiago (CREA). ${ }^{16}$

La planificación en el Ecuador se institucionaliza entre 1945 y 1965, en el marco de un debate que consideró la planificación nacional y regional como dos facetas de un mismo proceso; es decir, ninguna de las dos podía tratarse aisladamente sin perder el sentido de una planificación de conjunto. Sobre todo, la planificación regional era el instrumento que permitiría acercar la planificación nacional a las necesidades y potencialidades locales, porque era capaz de enfocar los problemas sociales, económicos y políticos de una comunidad y sus interrelaciones, dentro de un espacio físico local (Washima, 1981).

\section{Primeros estudios y propuestas de la JUNAPLA para la "regionalización" del Ecuador (1965 y 1968)}

Ecuador vive una etapa de inestabilidad política entre 1960 y 1973 que debilita la acción planificadora de la JUNAPLA, pero sin afectar el impulso a la promoción de estudios sobre las regiones económicas y la formulación del "Primer Plan General de Desarrollo Económico y Social 1964-1973"17 (JUNAPLA, 1963); pues estas debían ser soporte y marco teórico de referencia de la primera propuesta de regionalización. El antecedente de los primeros "esquemas de regionalización" del Ecuador, punto de partida para la realización de investigaciones más avanzadas sobre integración económica y reducción de desequilibrios regionales, fue el estudio que la JUNAPLA, bajo auspicios del Fondo Especial de Asistencia Técnica de Naciones Unidas, encargó en 1965 al economista alemán Hans Linnemann, y que fue por este completado en forma de "estudio preliminar" y bajo el título "Regiones económicas del Ecuador, su integración y desarrollo" (Linnemann, 1965). Aunque el estudio se fundamentaba en estimaciones estadísticas sujetas a un apreciable margen de error, se logró tener una primera descripción de las regiones económicas, especialmente su localización y la caracterización económica de las distintas áreas. Linnemann dividió el territorio nacional en 12 regiones económicas, y definió como "región económica" aquella "parte geográfica del país que tiene íntimamente bastantes características de unidad y similitud, pero que, al mismo tiempo, muestra marcadas diferencias con las partes que la circundan, en estructura y situación económica" (Linnemann, 1965, p. 9). La delimitación de las 12 regiones, resultante de la combinación de 5 factores (natural, humano, institucional, político-regional y económico), se basaba en las principales diferencias interregionales (figura 1).

El estudio reveló que las regiones económicas del país se hallaban dispersas, aisladas; que carecían de comunicación interna y externa, y que estas condiciones eran, entre otras, algunas de las causas del desigual desarrollo y distribución de ingreso del país. Sobre las bases de las anteriores consideraciones, Linnemann sugirió realizar nuevas investigaciones en tema de transporte y telecomunicaciones, organización del mercado interno, organización de un mercado de trabajo y desarrollo de la comunidad y, para el fomento de políticas públicas, recomendó promover la integración de la economía nacional ${ }^{18}$ y lograr un desarrollo regional balanceado ${ }^{19}$. Tres años después, a través del diario "El Comercio" (Planeación Regional para propender el Desarrollo Armónico e Integral del País, 1968, p. 17), la JUNAPLA informaba que adoptaría la planificación regional como estrategia para lograr un desarrollo armónico ${ }^{20}$ e Integral ${ }^{21}$. Con el fin de sentar las bases para la programación regional e implementarla, se modificó la estrategia prevista para la ejecución del Primer Plan Nacional General y se optó por una planificación que priorizara los problemas específicos de las diferentes regiones.

Para la propuesta de regionalización, conscientes de las limitaciones que imponía un análisis macroeconómico de los problemas del país, se utilizarían las escasas informaciones disponibles a nivel provincial, con la posibilidad de modificar los límites regionales propuestos una vez finalizadas algunas investigaciones. Una primera aproximación organizaba el país en cinco "regiones de planeamiento", clasificadas en 


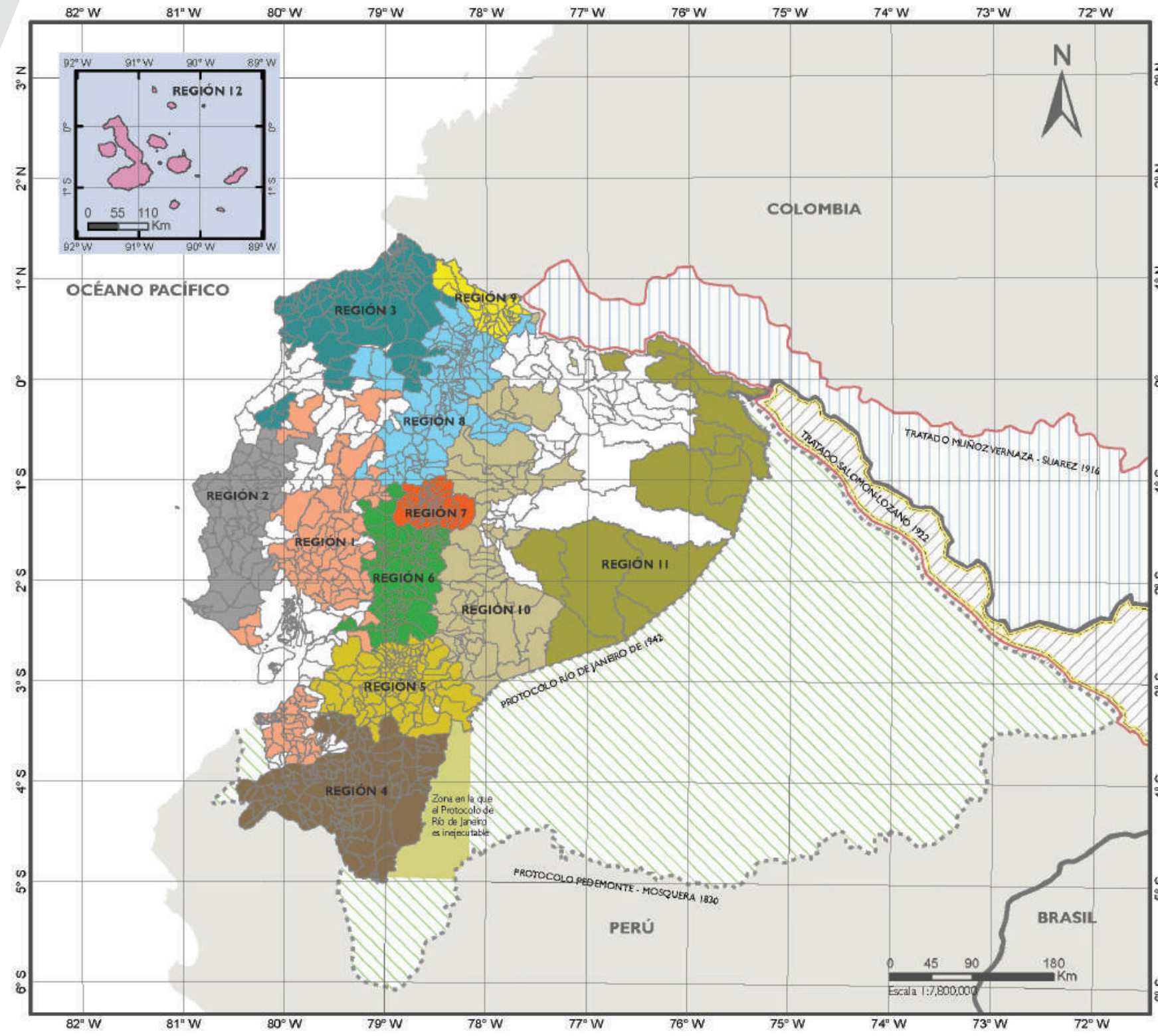

ROPUESTA DE HANS LINNEMANN DE LAS REGIONES ECONÓMICAS DEL ECUADOR (1965)

\section{FUENTES:}

Linneman, Hans. "Regiones económicas del Ecuado su integración y desarrollo. Estudio Preliminar".

Nacional de Planificación y Coordinación Económica, 1965, 28

\section{SIMBOLOGÍA}

Límites de Ecuador

Frontera Internacional

-."- Protocolo de Río de Janeiro 1942 (Ecuador-Perú)

Zona en la que el Protocolo de Río de Janeiro es inejecutable

Tratado Muñozvernaza-Suarez 1916 (Ecuador-Colombia)

Tratado Salomón-Lozano 1922 (Colombia-Perú)

\section{Regiones económicas}

Región I: Bolvarar Cañar, Pichincha, Manabí Los Ríos Guayas, El Oro, Cotopaxi

Región 2: Manabí, Guayas

Revón 3. En

Región 4: Loja, Zamora Chinchipe, El Oro

Región 5:Azuay, Morna Santiazo, Cañar

Región 6: Cañar, Chimborazo, Bolvivar, Cotopax

Región 7:Tungurahiua Cotopaxi

Región 8: Cotopaxi, Pichincha, Imbabura, Napo Pastaz

Regiớn 9: Carch

Región 10: Napo Pastaza

Región I : Napo Pastaza, Morona Santiago

Región I2:Archipiélago de Colón

\section{TUDIOY ELABORACIÓN}

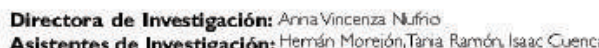

Fecha Redibujo: Julio 2015

MAPA I

Figura 1. Mapa: Regiones Económicas del Ecuador según estudio de Linnemann, 1965. Elaboración: @ Anna Vincenza Nufrio, Hernán Morejón, Tania Ramón, Isaac Cuenca.

Figura 2. Mapa: Propuesta de "Regionalización Horizontal" del Ecuador, JUNAPLA 1968

Elaboración: (C) Anna Vincenza Nufrio, Hernán

Moreión. Tania Ramón. Isaac Cuenca.

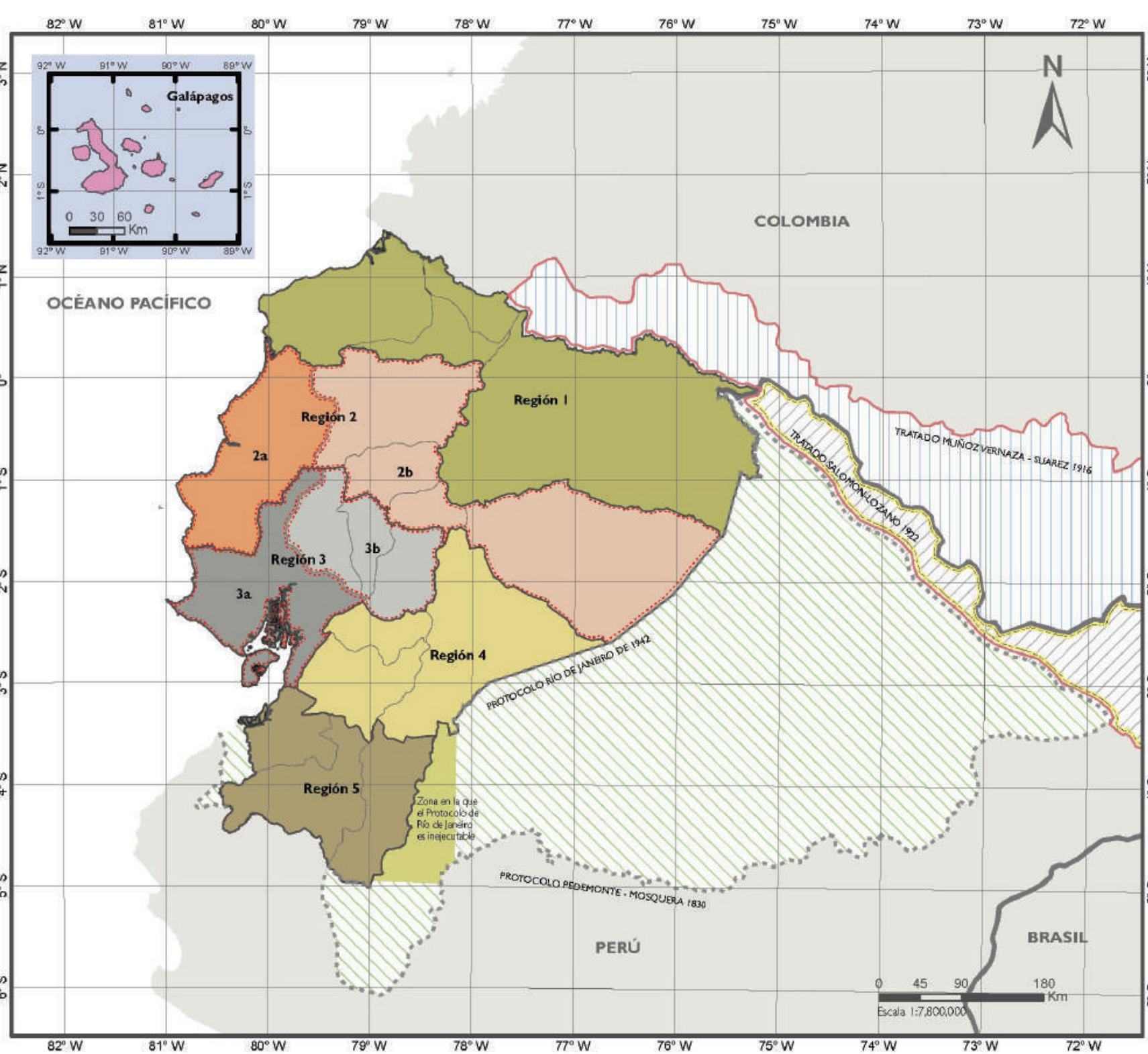

cinco grupos básicos según sus problemas específicos, y se fraccionaban ulteriormente algunas de ellas en espacios subregionales. La extensión de cada región se basaba en la búsqueda del equilibrio entre el tamaño de la población residente y su territorio representativo, razón por la cual, en el caso de las regiones 2 y 3 , se consideró oportuno, por su densidad poblacional, realizar una ulterior división en dos subregiones (Figura 2).

PROPUESTA DE LAJUNTA NACIONAL DE DEL ECUADOR, 1968

FUENTES:

"Planeación Regional para propender el Desarrollo Armónico e integral del Pais", El Comercio Quito), 12 de abril, 1968, 17

\section{SIMBOLOGía}

Límites de Ecuador

Frontera Internaciona

"'Protocolo de Rúo de laneiro 1942 (Ecuador-Perú) Zona en la que el Protocolo de Río de Janeiro s inejecutable

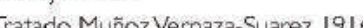
(Ecuador-Colombia)

U/ Tratado Salomón-Lozano 1922 (Colombia-Perí)

\section{Regiones y subregiones propuestas}

\section{Región I: Esmeraldas, Imbabura, Carchi y Napo}

Región 2:

Subregión 2a: Manabi

Subregión 2b: Pichincha. Cotopaxi,Tungurahua

Pastaza

Región 3

Subregión 3a: Guayas

Subregión 3b: Los Rios, Bolivar y Chimborazo

Región 4: Azuay, Cañar y Morona Santiago

Región 5: El Oro, Loja y Zamora Chinchipe 
Los criterios básicos para la regionalización, que fue del tipo "Regional Horizontal", se basaron en:

Promoción de la integración física, económica y social de las tres regiones naturales del país (Costa, Sierra y Oriente), especialmente para mejorar la utilización de los recursos.

Formación de "unidades de planeamiento" que reunieran mejores condiciones para la utilización de los recursos interprovinciales, mediante la agrupación de varias provincias.

Salvaguardia de la unidad de las provincias pertenecientes a una región y respeto de la organización administrativa existente.

Los objetivos principales fueron:

Determinar con precisión el espacio físico en el que se desenvolverían los programas de desarrollo.

Corregir los peligros de la centralización económico-administrativa.

Reducir las desigualdades a nivel provincial: descentralizando acciones y centralizando políticas y decisiones de carácter nacional.

Democratizar el proceso de planificación potenciando los contactos con organismos provinciales y grupos sociales y políticos locales.

Lograr una mayor eficacia operativa y administrativa en la ejecución de los planes.

Fomentar el desarrollo armónico e integral de la economía nacional.

El enfoque adoptado obligaba a introducir en la planificación del desarrollo la "variable espacial", constituyendo esta un aporte positivo para abordar los problemas que afectaban a una determinada comunidad, o a una región específica.

\section{El CREA y el fomento del Desarrollo Regional en las provincias de Azuay Cañar y Morona Santiago (1958-1979)}

El primer Plan General de Desarrollo Económico y Social 1963-1974 del Ecuador propició tres tipos de reformas: de la administración pública; tributaria; de la estructura de la tenencia de la tierra y la expansión de la frontera agrícola. La reorganización administrativa suprimió a los Consejos Provinciales y delegó ciertas funciones locales (promoción, programación y ejecución de programas de desarrollo regional) a los Organismos de Desarrollo Regional, para que estos operaran con cierta autonomía en sus propias jurisdicciones (Moncada, 1974).

Con la coordinación de acciones entre los ONP y los ODR, los Planes Nacionales de Desarrollo comienzan a "regionalizarse", encontrando en las provincias de Azuay, Cañar y Morona Santiago terreno fértil para la promoción y realización de proyectos pioneros para el país. El Centro de Reconversión Económica del Azuay, Cañar y Morona Santiago (CREA) inició oficialmente sus actividades en abril de 1959 dedicándose a impulsar el desarrollo regional en la Región Centro-Sur del Ecuador, además de realizar estudios e informes y encontrar respuestas a situaciones de emergencia en las zonas urbanorurales. Sus emprendimientos y acciones persiguieron la consecución de tres objetivos prioritarios:

1. Estudio, planificación y ejecución de programas de desarrollo regional en Azuay, Cañar y Morona Santiago.

2. Conservación del suelo, reforestación, utilización de fuentes de agua para riego, fomento agropecuario y colonización.

3. Promoción de nuevas industrias y regulación y conducción de las existentes.

Un periodo de madurez del CREA en tema de fomento de desarrollo regional inicia en enero de 1971, cuando su primer Administrador General, el Sr. Emmanuel Martínez Palacios, perpetuando la labor de promoción de estudios, entregó a la JUNAPLA 
el informe "Planteamientos para un Plan Cuatrienal de Desarrollo en las provincias de Azuay, Cañar y Morona Santiago" (Martínez, 1971), documento que destacaba el ahondamiento del desequilibrado desarrollo espacial del Ecuador, demostraba que dichas provincias carecían de los servicios más elementales para satisfacer las necesidades básicas de la población y, siguiendo los lineamientos planteados en el Plan Nacional 1964-1973, proponía la adopción de un modelo de desarrollo económico "armónico" e "integral". El Sr. Martínez entregaba a la JUNAPLA el documento definitivo de un informe preliminar redactado diez años antes, titulado "Lineamientos generales para un Plan de Desarrollo Integral del Azuay, Cañar y Morona Santiago" (Martínez, 1962).

Frente a los limitados recursos disponibles, las oportunidades que brindaban los programas de ayuda de cooperación internacional fueron vistas por Martínez como una posibilidad de acceso a una sustantiva ayuda financiera y a una más experta asesoría técnica. En la introducción del estudio se señalaba que en distintos países de la región crecía el interés en los Programas de Desarrollo Integral de largo alcance; que era importante la adhesión al "Programa Alianza para el Progreso" (ALPRO) porque este ofrecía "oportunidades nunca pensadas para poder ejecutar dichos planes" (Martínez, 1962, 1 y que el CREA debía aprovechar los medios que Estados Unidos ponía a disposición de aquellos países que con esfuerzo propio querían conseguir el progreso. Asimismo, añadía que era necesario beneficiarse de las técnicas y de los recursos económicos adecuados y que este puente sería "construido por el noble pueblo norteamericano, arquitecto mundial de la justicia y de elevados principios y realizaciones de la democracia" (Martínez, 1962, segunda página de la sección "Presentación") . Su anhelo por conseguir la ayuda de Estados Unidos significaba abrazar el credo de que en América del Sur existían las capacidades suficientes para afrontar y resolver los problemas, que las inversiones de cooperación internacional perseguían el acoplamiento de los Planes Nacionales a los Planes Regionales, y que este imperativo tenía una obligación: ser impulsado desde abajo, desde las propias regiones. Por consiguiente era necesario formular y llevar a la práctica el primer Programa Integral de Desarrollo Económico Regional del Ecuador, como un "Plan Piloto" o "Plan Experimental" para la definición de pautas de los propios Planes Nacionales. Con estos antecedentes, las bases para la formulación de un Plan Regional Integral para las provincias de Azuay, Cañar y Morona Santiago quedaban fijadas.

\section{La introducción del "enfoque espacial" en la Planificación Regional del Desarrollo (1974-1978)}

En febrero de 1972 el Gobierno Militar de las Fuerzas Armadas Revolucionarias tomó el poder en el Ecuador, coincidiendo el inicio de su gestión con el "boom petrolero" y un incremento notable del presupuesto estatal. La bonanza económica fue propicia a la actividad de promoción de estudios de la JUNAPLA que, en 1972, publicaba dos nuevos documentos: "Filosofía y Plan de Acción del Gobierno Revolucionario y Nacionalista del Ecuador" (JUNAPLA, 1972b) y el "Plan Integral de Transformación y Desarrollo 19731977" (JUNAPLA, 1972a). Iniciando la década de los 70s, el enfoque global-sectorial, aplicado durante los primeros 20 años de actividad planificadora en Ecuador, resultó inapropiado para captar ciertos problemas locales. A tal propósito, y de acuerdo a los enunciados del Plan Integral, la División de Estudios Regionales de la JUNAPLA impulsó la planificación con un "enfoque regional", a manera de puente entre la planificación nacional y las necesidades y potencialidades locales: realizó investigaciones sobre las problemáticas regionales para establecer un conjunto coherente de medidas de política económica regional; definió un conjunto coherente de objetivos económicos sub-nacionales; esbozó una estrategia regional-nacional como guía para las grandes líneas de acción a seguir; y estableció pautas y directivas para localizar proyectos de inversión de carácter estratégico.

Indudablemente, "localizar proyectos" significaba definir una "estrategia espacial" de desarrollo y, para ello, la JUNAPLA promovió entre 1975 y 1977 un conjunto de investigaciones orientadas a formular una nueva propuesta de regionalización, y que fueron integralmente publicadas en el Atlas "Planificación Regional: Estructura del 
22 Las notas habían sido redactadas entre abril y julio de 1975, en ocasión del "Curso de Planificación Regional del Desarrollo" dictado en Santiago de Chile y organizado bajo auspicio del ILPES, la CEPAL y el PNUD.

\section{Un modelo gravitatorio formaliza} estudia y prevé la geografía de los flujos o de las interacciones.
Espacio Ecuatoriano" (JUNAPLA, 1977). Con este estudio, la "variable espacial" fue finalmente incorporada a las políticas de desarrollo regional y fue entendida como un elemento de análisis complementario a las variables económica y social. El enfoque espacial adoptado sintetizaba, en un amplio conjunto de variables, los procesos de ocupación territorial, la organización y desarrollo del espacio socioeconómico y la apropiación y aprovechamiento de los respectivos recursos naturales, humanos, económicos y políticos, permitiendo conocer finalmente la estructura espacial del país y su incidencia en el proceso general del desarrollo. Parte del estudio (mapas y métodos de análisis) se fundamentaba en las notas técnicas de Henri Meót, asesor regional de la CEPAL en planificación regional, redactadas durante las misiones de asesoría realizadas en Ecuador en 1974. Las notas, tituladas "El Concepto de Región. Documentos Complementarios" (Meót, 1975a) y "Diagnostico Espacial del Ecuador: Método de diseño de las zonas de polarización de los centros poblados" (Meót, 1975b) representaban el diagnóstico espacial del país y planteaban una estrategia espacial de desarrollo como etapa clave del proceso de planificación. ${ }^{22}$

Para Meót, el diagnóstico espacial de un universo socio-económico determinado debía ser "el estudio crítico e interpretativo de todo el espacio, de las características tendencias y posibilidades de los sistemas de distribución sub-nacional de los recursos, procesos y beneficios del desarrollo" (1975b p. 1). Sobre todo, las zonas polarizadas de los principales centros poblados, tenían suma importancia a la hora de entender las interrelaciones estructurales existentes entre los centros y sus zonas de influencia, pues estas permitirían conocer el sistema de ciudades vigentes, el "funcionamiento" del espacio, y la localización del conjunto de unidades socioeconómicas y territoriales, generalmente poco perceptibles si observadas a escala nacional. Además, las interrelaciones existentes entre estas estructuras permitirían tener una visión dinámica del espacio; es decir, conocer el funcionamiento espacial. La dinámica espacial, estudiada a través de la observación de las interrelaciones "intrazonales" e "interzonales", junto a la aplicación de un modelo gravitatorio ${ }^{23}$, permitiría diseñar las zonas de polarización de los principales centros poblados del país. Finalmente, la síntesis de las correlaciones existentes entre las estructuras espaciales de constitución (zonas homogéneas) y el análisis dinámico de interrelaciones (zonas polarizadas) permitiría tener la visión definitiva de la estructura del espacio nacional (figura 3).

\section{Ecuatoriano, JUNAPLA 1976.}

Figura 3. Mapa: Estructura del Espacio Elaboración: @Anna Vincenza Nufrio, Hernán Morejón, Tania Ramón, Isaac Cuenca.

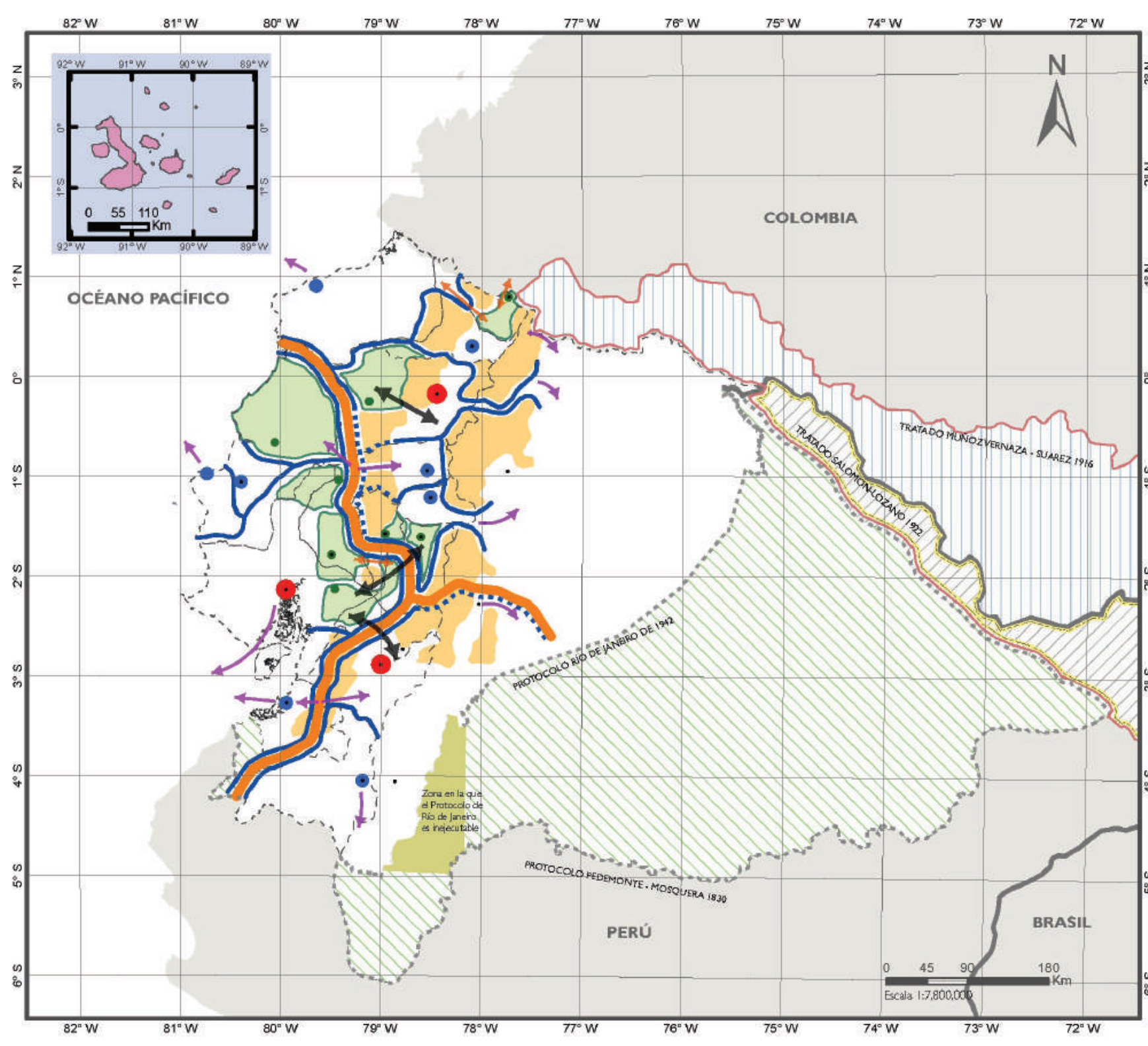

ESTRUCTURA DEL ESPACIO ECUATORIANO, JUNAPLA, 1976

FUENTES:

Ecuador:Junta Nacional de Planificación y Coordinación Económica. Planificación Regional: Estructura del Espás Ecuatoriano. Guayaquil: A.G. Senefelder, 1977, 179.

SIMBOLOGÍA

Limites Interncionales e Internos de Ecuador Frontera Intemacional

-1.." Protocolo de Río de Janeiro 1942 (Ecuador-Penú) Zona en la que el Protocolo de Río de laneiro es inejecutable (1916 (Ecuador-Colombia)

VI, Tratado Salomón-Lozano 1922 (Colombia-Perú)

Estructura del Espacio Ecuatorian

Centro de ler nivel

Centro de 2do nive

- Centro de 3ernivel

Límite del sistema

L Límite de subsistema

- Límite de zona de polarización

Area de preservación nacional

Zona de transición

$\longrightarrow$ Expansión

$\leftrightarrow$ Intercomunicación principal

$\longleftrightarrow$ Intercomunicación secundaria

ESTUDIOY ELABORACIÓN

Directora de Investigación: Ama Vincenza Nufrio 
$24 \quad$ Cada centro tiene sus respectivos subsistemas y zonas polarizadas y existe un sistema de intercomunicación principal y secundario entre la Costa, la Sierra y en las zonas fronterizas.
El Atlas recogía las notas metodológicas de Meót, reproduciendo fielmente algunos de sus mapas de estudio: el "sistema de regiones", las "unidades geoeconómicas subnacionales" y los esquemas gráficos que mostraban cómo el territorio ecuatoriano se organizaba en tres áreas principales que tenían en Quito, Guayaquil y Cuenca los centros urbanos más significativos ${ }^{24}$. El estudio tuvo particularmente en cuenta la viabilidad práctica de la propuesta de regionalización: confrontó el esquema estructural de la realidad socio-económica-espacial con la división político-administrativa del país (a nivel cantonal y provincial) y consideró la zonificación administrativa existente (ministerios, organismos adscritos y organismos de desarrollo regional) como otro referente de interés (Ojeda, 2000).

Sobre la base de estos estudios la JUNAPLA formuló en 1976 una segunda propuesta de regionalización planteando la división del territorio nacional en ocho regiones, e individualizando "varios sistemas regionales", cada uno con un grado de especialización económica distinta (figura 4).

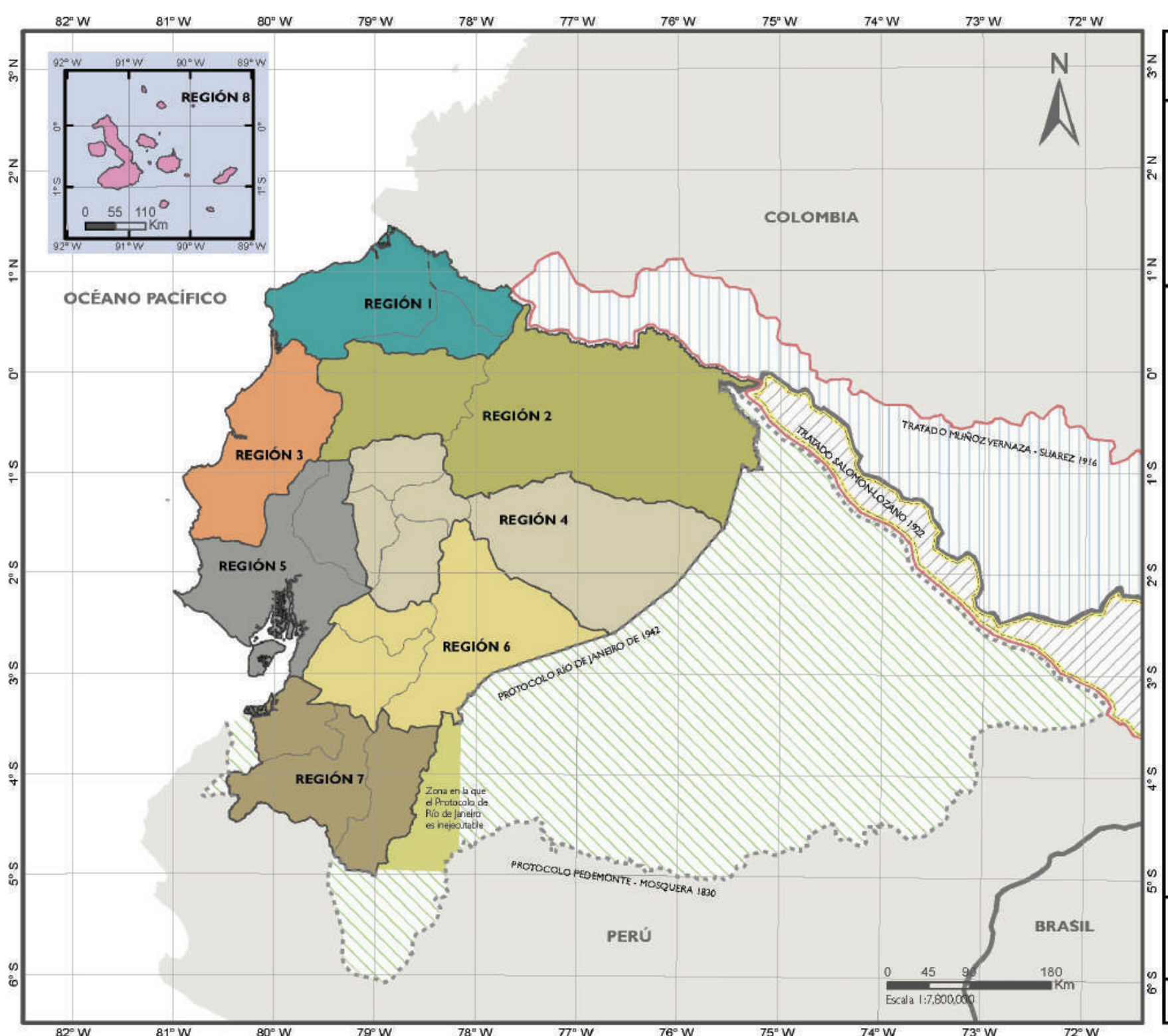

Figura 4. Mapa: Propuesta de Regionalización Horizontal del Ecuador, JUNAPLA 1976.

Elaboración: OAnna Vincenza Nufrio, Hernán Morejón, Tania Ramón, Isaac Cuenca.

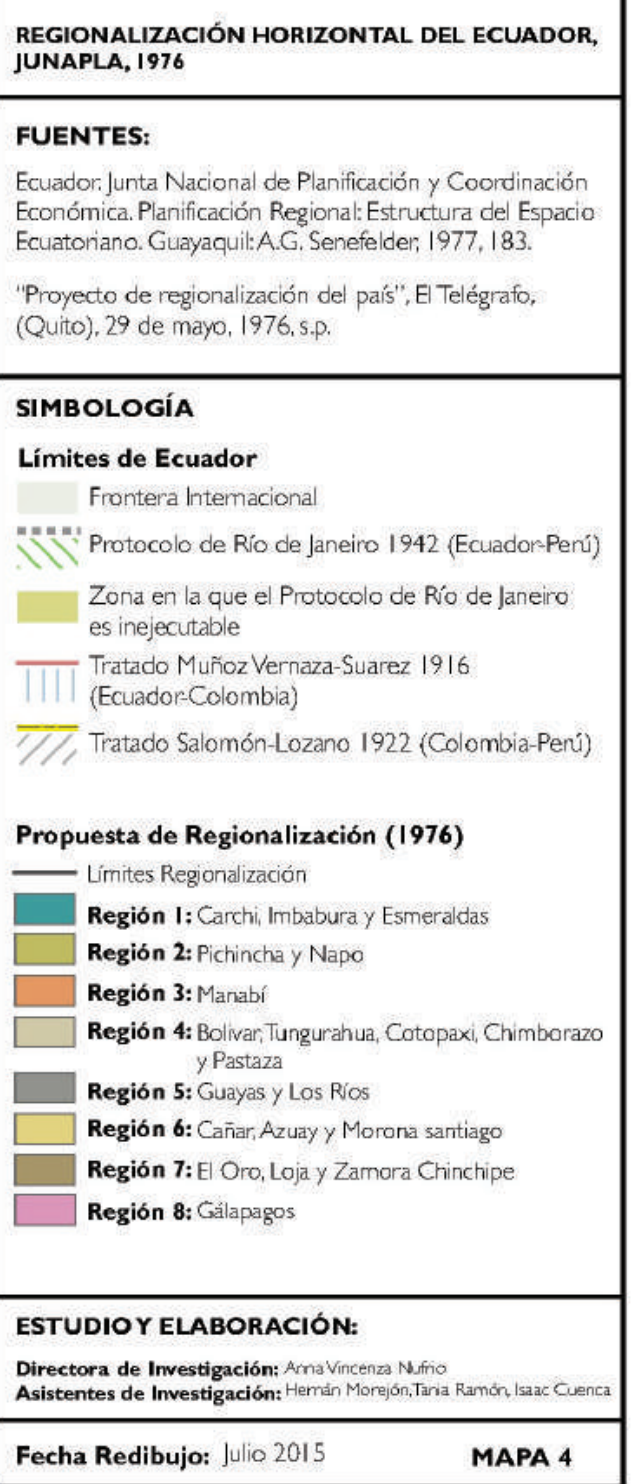

El principio de "horizontalidad" regía nuevamente la propuesta, la cual se fundamentaba en la obligada integración de dos o tres regiones, sobre la base del grado de coincidencia de las zonificaciones de carácter administrativo, técnico y jurídico, establecidas por diversas instituciones del Estado. Hablar de planificación regional no significaba, en estos momentos, reorganizar el país según una nueva división espacial; al contrario, se hacía necesario un entendimiento profundo sobre la estructuración del espacio físico nacional, y de cómo el desarrollo tenía que acontecer en él. En consecuencia, estudiar con rigor las interrelaciones geográficas y sociales de cada zona económica, enriqueciendo la lectura con el estudio de flujos de bienes, servicios y personas, iba a ser la segunda etapa de un trabajo que se vio claramente reflejado en las propuestas de planificación que, desde el CREA, se formularon a finales de los años 70, en pro de la Región Centro-Sur del Ecuador. 


\section{Génesis del Primer Plan Integral de Desarrollo Regional de las Provincias de Azuay, Cañar y Morona Santiago (1978-1982)}

El CREA fue el ODR que, de acuerdo a los principios del documento "Filosofía y Plan de Acción del Gobierno Revolucionario y Nacionalista del Ecuador" (JUNAPLA, 1972b), encauzó con premura acciones dirigidas a reducir los desequilibrios económicos y sociales en la Región Centro-Sur. El 24 de agosto de 1974, con el nombramiento del nuevo Administrador y Director Ejecutivo, el Mayor José Gallardo Román, el CREA pasó a ser dirigido por representantes del Gobierno Revolucionario Nacionalista de las Fuerzas Armadas, en un momento de logros y éxitos de su institución ${ }^{25}$. El 5 de septiembre de 1975, teniendo como referencia los planteamientos del Plan Integral de Transformación y Desarrollo 1973-1977, la JUNAPLA y el CREA, emprendieron un proceso de planificación regional coordinado, registrado en el marco del Convenio de Asistencia Técnica de Naciones Unidas ECU/74/005, con el fin de formular el Primer Plan de Desarrollo Regional Integral para las provincias de Azuay, Cañar y Morona Santiago. JUNAPLA y Naciones Unidas se comprometían a prestar apoyo técnico al CREA y a nombrar un coordinador que cumpliría las funciones de enlace permanente entre las dos instituciones. El CREA tomaba la responsabilidad de constituirse en la unidad básica de realización del proyecto y coordinaría acciones con las otras instituciones regionales participantes. ${ }^{26}$

Mientras se preparaba el "Documento de Pre-Diagnostico ${ }^{27}$ de la realidad regional, el Mayor José Gallardo Román renunciaba al cargo de Director Ejecutivo del CREA, siendo remplazado el 4 de mayo de 1976 por el Mayor Francisco Moncayo Gallegos y coincidiendo su llegada con la publicación del estudio "Plan de Desarrollo Regional Integral para las Provincias de Azuay, Cañar y Morona Santiago. Introducción" (CREA, 1976). En la presentación pública del Pre-Diagnostico del Plan Regional Integral, el Mayor Moncayo destacó la importancia de la planificación regional integral para una adecuada asignación de recursos, pues esta permitiría concretar objetivos sociales, económicos y biológicos de interés particular para cada región.

El economista Oswaldo Washima Chávez, director del Departamento de Planificación del CREA, dirigió los estudios del Plan Integral ${ }^{28}$ enfocándolos en torno a dos aspectos principales: el análisis de la estructura espacial-sectorial de la región y la lectura de la coyuntura existente en 1976. El objetivo principal consistía en elevar el nivel de ingresos de las capas sociales más bajas mediante una reorientación del uso de la tierra en las provincias de Azuay y Cañar (integrando los minifundios) y fomentando un uso intensivo de la tierra en la provincia de Morona Santiago (con la colonización semidirigida). Los objetivos específicos, de largo plazo, tenían entre sus metas: proporcionar a los sectores sociales mayoritarios el acceso a bienes y servicios básicos; fortalecer el aparato productivo para una adecuada interacción entre economía, proceso social y medio ambiente; robustecer el rol de la región en el contexto nacional, asegurando

25 Recordamos el Proyecto Hidroeléctrico Paute; el Parque Industrial de Cuenca; la creación del Instituto Panamericano de Artesanías y la redacción del Plan Quinquenal del Consejo Provincial y el Consejo Cantonal.

26 Gobernaciones, consejos provinciales, municipalidades, consejos cantonales, delegaciones de los ministerios y otras entidades, universidades, colegios profesionales, agrupaciones empresariales y clasistas, entre otros.

27 El Pre-Diagnóstico se componía de 6 documentos: Diagnóstico, Fijación de objetivos, Elaboración de una Estrategia de Desarrollo, Identificación de Programas y Proyectos, Implementación y establecimiento de Mecanismos de Control, y Evaluación del Plan.

28 En el estudio y asesoría externa colaboraron Jaime Idrobo y Raúl Paredes como contraparte nacional, y René Saa-Vidal y Hernán Latorre como contraparte internacional. su adecuada integración y participación en el proceso de desarrollo nacional; y alcanzar la participación consciente y crítica de la población en el proceso de desarrollo regional (JUNAPLA, ONU y CREA 1977). Identificar los principales factores de atraso de una zona respecto a otra, así como definir zonas económicas homogéneas, eran las acciones a emprender para poder definir "regiones de planificación" coincidentes con el límite político administrativo provincial. En enero de 1977 el CREA y la División de Estudios Regionales de la JUNAPLA, siempre bajo el auspicio del Proyecto "ECU/74/005", publican un nuevo estudio complementario al Plan Regional: "Áreas Básicas de Planificación: Enfoque para un análisis desagregado. La experiencia en la Región del Azuay, Cañar y Morona Santiago, Ecuador" (JUNAPLA, ONU y CREA, 1977). En este estudio el tema de la "descentralización de las decisiones" adquiría un sentido especial, en cuanto el análisis de la realidad local y la formulación de las propuestas de acción del Plan Regional solo podían ser operativas si eran promovidas desde el nivel regional y local. La aplicación de métodos participativos contaba ya con un documento publicado con anterioridad por la JUNAPLA en diciembre 1976, titulado: "Bases para la implementación de un sistema de participación de la población en el proceso de Planificación Regional del Ecuador" (Rusque, 1976). Para lograr la participación de la población y concretar las instancias y niveles desde donde generar 
29 Por ser la parroquia rural el sistema administrativo más pequeño, era más fácil fomentar a esta escala la participación de las organizaciones institucionales de base desde el principio.

Figura 5. Mapa: Plan Integral de Desarrollo Regional de las Provincias de Azuay, Cañar y Morona Santiago (1977-1982).

Elaboración: @Anna Vincenza Nufrio, Hernán Morejón, Tania Ramón, Isaac Cuenca. las acciones participativas, se decidió delimitar el territorio en treinta Áreas Básicas de Planificación (ABP), cada una de ellas correspondiente al nivel administrativo de la parroquia rura ${ }^{29}$, y caracterizadas por problemáticas, limitaciones y potencialidades específicas (figura 5). Una ABP fue definida como un espacio geográfico de carácter unitario, un área homogénea donde se encuentra asentado un grupo humano, cuyos individuos están ligados por relaciones históricas de producción específicas y que representan, a su vez, las estructuras económicas en el espacio (Idrobo, 1977). Áreas que se interrelacionan formando sistemas y subsistemas productivos funcionales, interdependientes; territorios contiguos en los que se localizan agentes o medios involucrados en un mismo tipo de relaciones, como en el caso de áreas de producción campesina, o que poseen un mismo nivel de calidad de atributos, por ejemplo, áreas ocupadas por ciertos grupos étnicos, áreas con familias con un ingreso anual similar, 0 áreas con producciones dominantes similares.

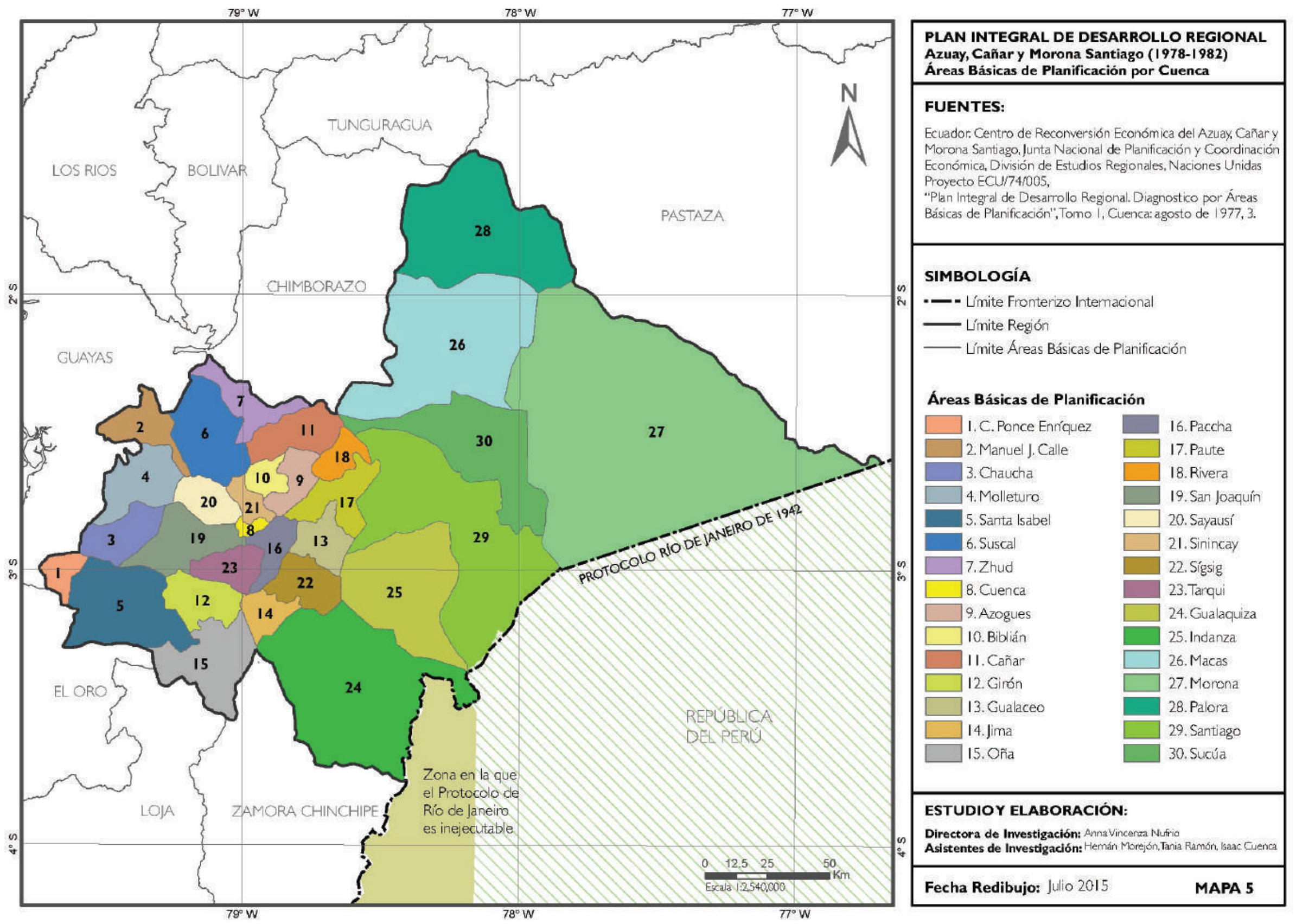

30 Las primeras experiencias de DRI fueron ensayadas en Israel, desde su independencia en 1948 y hasta 1984 y fueron planeadas y establecidas por el profesor Raanan Weitz y su equipo permitiendo efectuar la transición desde la agricultura de subsistencia a la agricultura de alta tecnología orientada a la exportación.

\section{$31 \quad$ Ministerio de Agricultura del Ecuador.}

32 Instituto Nacional de Recursos Hidráulicos del Ecuador.

33 Con excepción de los servicios de crédito, que por su propia naturaleza debían ser atendidos por un banco, y algunas obras de infraestructura encargadas a empresas especializadas.
Las ABP fueron el referente para especificar acciones prioritarias y experiencias piloto a implementar y que, en línea con cuanto ocurría en otros países latinoamericanos, se formalizaron a través de Proyectos de Desarrollo Rural Integral (DRI) ${ }^{30}$. Estos eran proyectos pensados como estrategias para llevar a cabo acciones integrales en el sector agropecuario: el DRI de Santa Isabel y el DRI de Cañar-Suscal-Zhud. Dichas estrategias, que por lo general estuvieron integradas por los técnicos de las diferentes entidades participantes (CREA, MA ${ }^{31}$, INERH ${ }^{32}$, entre otros) y dependieron técnica y administrativamente de un jefe de unidad $^{33}$, se fundamentaron en los siguientes conceptos básicos:

El desarrollo rural debía ser el resultado de la coordinación, a nivel regional, de los objetivos planteados por las políticas nacionales y de las necesidades particulares de cada una de las unidades locales de producción, siendo la región el escenario donde se desenvuelve la acción del DRI.

El crecimiento del sector agrícola constituía la clave del desarrollo rural, y su fomento exigía el desarrollo simultáneo de todos los sectores de la economía. 
El desarrollo incluía aspectos sociales, económicos, físicos e institucionales y, por lo tanto, la planificación debía referirse a ellos en forma concomitante.

Sin embargo, algunas limitaciones dificultaron su alcance y éxito, impidiendo que a través de su plena realización se ejecutara también parte del Plan Integral Regional:

LosDRI tuvieron un carácterasistencialista. Funcionaron con un enfoque "dearriba hacia abajo" y sin ninguna participación masiva de los auténticos promotores del desarrollo rural: los campesinos. Recursos humanos y económicos, destinados a los proyectos, fueron utilizados de manera inapropiada, y tan solo un pequeño grupo de campesinos, casi siempre los representantes de las comunidades, pudo beneficiarse de las ayudas.

Siendo la coordinación interinstitucional un objetivo instrumental para la realización de los DRI, esta no se plasmó en ningún mecanismo permanente, y la falta de integralidad y de coordinación entre las instituciones involucradas demostró que los problemas fueron tratados aisladamente. Recordamos que distintas instituciones operaban en cada $A B P$, porque eran responsables del desarrollo regional. ${ }^{34}$

No existió una unidad de monitoreo encargada de vigilar y evaluar de manera permanente los resultados parciales, y que incluyera a los beneficiarios de los DRI: los campesinos. Es notoria la debilidad existente, en el caso de Ecuador, del diseño y aplicación de sistemas de seguimiento y evaluación de los DRI; las informaciones disponibles permiten afirmar que la toma de decisiones correctivas de estrategias DRI se sustentó en información parcial y de tipo coyuntural, lo que explica en parte las desviaciones observadas durante la ejecución de los programas.

El limitado potencial agroeconómico de la mayor parte de las ABP no fue siempre suficientemente atractivo para los organismos de financiamiento externo; sobre todo porque la extensión de las áreas, unida a los bajos índices de equipamiento y la falta de vías de acceso (vías principales y caminos vecinales) impidió la comunicación y el intercambio entre las distintas ABP y determinó que su producción se basara en el autoconsumo y fuese poco orientada al mercado.

La falta de estudios de factibilidad dificultó la negociación financiera de los proyectos, retrasando o impidiendo su ejecución.

Las acciones encaminadas a ejecutar parte de los lineamientos planteados en el Plan Integral Regional, a través de las estrategias DRI, se estancaron en 1979 cuando la JUNAPLA fue substituida por el Consejo Nacional de Desarrollo (CONADE) por una reforma constitucional y, paralelamente, un cambio en la Dirección ejecutiva del CREA inauguró una etapa de inestabilidad de la Institución.

No obstante a partir de 1980, CONADE, a través de la Secretaría de Desarrollo Rural Integral (SEDRI) promueve la creación del subsistema de desarrollo rural integral dentro del sistema nacional de la Administración Pública, como un mecanismo para la utilización eficiente de recursos humanos del estado, y con Decreto No. 514 asigna a SEDRI funciones de planificación, programación, organización, coordinación y ejecución de proyectos DRI; los primeros años 80 marcaron en Latinoamérica, así como en Ecuador, un retroceso de la Planificación como instrumento de política pública.

$34 \quad$ Ministerio de Agricultura (MAG), Centro de Reconversión Económica del Azuay, Cañar y Morona Santiago (CREA), Instituto Ecuatoriano de Recursos Hidráulicos (INHERI), Instituto Ecuatoriano de Obras Sanitarias (IEOS), Centro Ecuatoriano de Servicio Agrícola (CESA), Instituto Ecuatoriano de Reforma agraria y Colonización (IERAC), Dirección de Educación, Consejo Provincial, Consejo Cantonal, Banco de Fomento, Colegios Técnicos, Universidades, etc.
Se desmantelaron muchos ONP cuando no se redujeron sus funciones y sus ámbitos de influencia, bajo la premisa de que el mercado se haría cargo de reorientar y hacer más eficiente el proceso de desarrollo. El Estado cumplió, desde este momento, un papel subsidiario, funcional a la preponderancia que adquirirían el sector privado y el mercado (Máttar, 2013; Moncayo, 2013). En el caso particular de las políticas regionales, estas fueron reemplazadas por un proceso de descentralización de los recursos fiscales, con acento marcadamente municipalista. 


\section{Conclusiones}

A lo largo de la investigación se pudo observar cómo la historia de la planificación regional en el Ecuador, entre 1952 y 1982, se basó en la coordinación interinstitucional entre un Organismo Nacional de Planificación (JUNAPLA), los Organismos de Desarrollo Regional que operaron en distintas regiones del país, entre los que destaca el CREA, y las Entidades de Cooperación Internacional al Desarrollo (NACIONES UNIDAS, ILDIS, ILPES, BID, CAF, AID, entre otros).

La práctica de la planificación nacional y regional aplicada en Ecuador entre 1952 y 1982 ha tenido las siguientes características:

Las primeras experiencias de planificación nacional tuvieron un importante contenido sectorial (transportes, energía, desarrollo industrial o agrario) evolucionando sucesivamente hacia perspectivas propiamente territoriales, con connotaciones multisectoriales.

A mediados de los años 70, la planificación nacional adoptó un enfoque socioeconómico integral y se introdujeron la variable regional y la dimensión espacial. El espacio fue considerado como otro de los recursos puestos al servicio del desarrollo regional, y la planificación regional integral se caracterizó como una planificación regional urbano-rural, pues este sector representaba un área propicia para identificar y ejecutar proyectos, en virtud de sus particulares características socioeconómicas.

El enfoque "integral" permitió revelar que la formación económica y social de un territorio es como un todo orgánicamente integrado y articulado, y que solo la participación activa de toda la población, en la generación y beneficio de la riqueza, puede determinar el crecimiento económico y el desarrollo social.

- De manera decisiva, entre 1973 y 1979 se potenciaron estudios, investigaciones e informes técnicos; se formaron e intercambiaron expertos a nivel nacional e internacional; se fomentó el diseño de políticas y estrategias "a medida" de las diferentes regiones y en nombre de un desarrollo económico, social y espacial equitativo.

Con la generalización de las prácticas regionales se priorizaron regiones significativas, es decir, se delimitó una "región problema" y se elaboraron proposiciones tendientes a resolver "el problema singular" de dicha región.

EI CREA, que inicialmente fue creado como un centro de apoyo técnico para la ejecución de los Planes Nacionales a escala regional, se convirtió rápidamente en un centro técnico y administrativo con una cierta autonomía regional y en un referente nacional para los otros ODR que empezaban a operar en otras regiones del país. Enfrentó desafíos, promovió un sinnúmero de estudios e investigaciones, priorizó necesidades, definió objetivos clave para el desarrollo regional e invirtió recursos en aquellos proyectos dinamizadores para el desarrollo económico-social de la Región Centro-Sur.

El "Primer Plan Integral de Desarrollo Regional de las Provincias de Azuay, Cañar y Morona Santiago 1978-1982" se constituyó en el instrumento directriz, no solo de las actividades del CREA, sino en general de las acciones, programas y proyectos del Gobierno central.

Desde un punto de vista conceptual y metodológico, el Diagnóstico por Áreas Básicas de Planificación fue un instrumento eficaz para jerarquizar espacialmente las prioridades de una región y sustentar la tarea del desarrollo en los propios grupos humanos. Además, permitió iniciar algunos proyectos pilotos de Desarrollo Rural Integral (DRI) en la Provincias de Azuay y Cañar, aunque estos no lograron llegar a ejecutarse en su totalidad.

La falta de apoyo político continuado del Poder Central al Plan Integral Regional, la escasa asignación de recursos financieros, la confusa, cuando no ausente, coordinación interinstitucional, significaron poderosos y a veces insuperables impedimentos para la plena ejecución de lo programado y una clara desvalorización del Plan a nivel nacional. 
$35 \quad$ Las circunscripciones territoriales (CT) pueden ser conformadas por los pueblos indígenas, afro ecuatorianos y montubios.
Desde inicios de 2006, con las transformaciones político-económicas impulsadas por el Gobierno de Rafael Correa, la Planificación en el Ecuador se ancla nuevamente a las estrategias nacionales de desarrollo. El nuevo Ordenamiento Territorial ecuatoriano, consagrado en la "Constitución de Montecristi" (Asamblea Nacional Constituyente de Ecuador, 2008), en el Plan Nacional de Desarrollo para el Buen Vivir (República del Ecuador, 2017) y en el Código Orgánico de Organización Territorial, Autonomía y Descentralización (Asamblea Nacional Constituyente de Ecuador, 2010) se inscribe en un marco de reformas estructurales que incluye, con prioridad, la reivindicación de los derechos de las naciones y pueblos indígenas originarios; recupera el papel del Estado como promotor de un desarrollo que promueve la cohesión y la equidad interterritorial, y anima un debate en el cual el nuevo modelo de desarrollo reconoce, además del municipal, niveles territoriales intermedios como el regional y las circunscripciones territoriales (CT). ${ }^{35}$

En teoría, la nueva organización territorial prevé un amplio régimen de participación popular que incluye a comunidades, pueblos y nacionalidades; todos elementos que predisponen a que el debate sobre la regionalización vuelva a tener protagonismo. En la práctica, la actual Constitución recoge viejas aspiraciones y debates nacionales sobre la cuestión regional; sin embargo, su difícil implementación muestra cómo el modelo centralista del actual Gobierno marca un salto atrás en la historia de la descentralización y autonomía del país.

El mandato constitucional queda, en este momento, sin espacio real para su aplicación y la existencia de un marco jurídico favorable a la creación de regiones autónomas permanece anclada a la incertidumbre de su posible aplicación. El debate sobre la regionalización del Ecuador debe ser actualizado en el marco de las aspiraciones recogidas en la actual Constitución, y la planificación regional solo puede ser un acto democrático de reafirmación de las identidades territoriales y un ejercicio de civilización en pro del empoderamiento de las organizaciones sociales y comunitarias.

\section{Referencias Bibliográficas}

Asamblea Nacional Constituyente de Ecuador. (1945). Constitución Política de La República del Ecuador. Quito: Talleres Gráficos Nacionales.

Asamblea Nacional Constituyente de Ecuador. (2008). Constitución Política de La República del Ecuador. Quito: Asamblea Constituyente. Disponible en <http:// www.oas.org/juridico/pdfs/mesicic4_ecu_const.PDF>

Asamblea Nacional Constituyente de Ecuador. (2010). Código Orgánico de Organización Territorial, Autonomía y Descentralización. Quito: Ministerio de Coordinación de la Política y Gobiernos Autónomos Descentralizados. Disponible en

<http://www.oas.org/juridico/pdfs/mesicic4_ecu_org.pdf>

CREA. (1976). Plan de Desarrollo Regional Integral para las Provincias de Azuay, Cañar y Morona Santiago. Introducción. (Documento inédito). Cuenca, Ecuador.

Disponible en: Archivo del Centro de Reconversión económica del Azuay, Cañar y Morona Santiago, Sede Senplades Zona 6-Austro, Cuenca.

Galo, S. (1979). La Junta Nacional de Planificación y sus responsabilidades en el Desarrollo Económico y Social del Ecuador (pp. 23-44). En Salgado, G. (Ed.), 25 Años de Planificación Quito: Junta Nacional de Planificación. <http://repositorio. iaen.edu.ec/handle/24000/572>

Idrobo, J., et al. (1977). Áreas Básicas de Planificación: Enfoque para un análisis espacial desagregado. SIAP: Revistas Interamericana de Planificación, XI(44), 131-157.

JUNAPLA. (1956). Azuay y Cañar: Desarrollo Económico. Situación Agraria Forestal Quito: Casa de la Cultura Ecuatoriana.

Disponible en: Archivo del Centro de Reconversión económica del Azuay, Cañar y Morona Santiago, Sede Senplades Zona 6-Austro, Cuenca. 
JUNAPLA. (1963). Plan General de Desarrollo Económico y Social (1964-1973). Quito: Junta Nacional de Planificación y Coordinación Económica. <http://repositorio. bce.ec:8080/handle/32000/1578>

JUNAPLA. (1972a). Plan Integral de Transformación y Desarrollo. Resumen General. Quito: Junta Nacional de Planificación y Coordinación Económica.

http://repositorio.bce.ec:8080/handle/32000/1579

JUNAPLA. (1972b). Filosofía y Plan deAcción del Gobierno Revolucionario y Nacionalista del Ecuador - Lineamientos Generales. Quito: Gobierno Revolucionario Nacionalista de las Fuerzas Armadas.

Disponible en: Archivo del Centro de Reconversión económica del Azuay, Cañar y Morona Santiago, Sede Senplades Zona 6-Austro, Cuenca.

JUNAPLA. (1977). Planificación Regional: Estructura del Espacio Ecuatoriano. Guayaquil: A.G. Senefelder.

JUNAPLA y OEA. (1978). Memoria. Seminario Taller sobre formulación del Programa de Desarrollo Local (Rural Urbano Integrado) de Cañar-Suscal-Zhud y Santa Isabel. Cuenca, Cañar, Santa Isabel: JUNAPLA y OEA.

Disponible en: Archivo del Centro de Reconversión económica del Azuay, Cañar y Morona Santiago, Sede Senplades Zona 6-Austro, Cuenca.

JUNAPLA, ONU y CREA. (1977). Áreas Básicas de Planificación: Enfoque para un análisis espacial desagregado. La experiencia en la Región del Azuay, Cañar y Morona-Santiago-Ecuador. Quito: Oficina de Publicaciones.

Disponible en: Archivo del Centro de Reconversión económica del Azuay, Cañar y Morona Santiago, Sede Senplades Zona 6-Austro, Cuenca.

Linnemann, H. (1965). Regiones económicas del Ecuador, su integración y desarrollo. Estudio Preliminar. Quito: Junta Nacional de Planificación y Coordinación Económica. Disponible en <http://repositorio.iaen.edu.ec/handle/24000/2658>

Martner, R. y Máttar, J. (Comp.) (2012). Los fundamentos de la planificación del desarrollo en América Latina y el Caribe. Textos seleccionados del ILPES (19621972)

Santiago de Chile:CEPAL/ILPES. Disponible en

<http://repositorio.cepal.org/bitstream/handle/11362/2621/S2012845_es.pdf?sequence=1>

Máttar, J. (Ed.). (2013). Planificación para el desarrollo y la integración regional. Propuesta estratégica de mediano plazo para la cooperación del ILPES con los Países de América Latina y el Caribe. Brasilia: ILPES. Disponible en

<https://www.cepal.org/crp-ilpes/noticias/documentosdetrabajo/5/51585/2013-946_ CRP.14_Propuesta_estrategica_ESP-WEB.pdf>

Martínez P., E. (1962). Lineamientos generales para un Plan de Desarrollo Integral del Azuay, Cañar y Morona Santiago [Informe inédito del CREA]. Cuenca, Ecuador.

Martínez P., E. (1971). Planteamientos para un Plan Cuatrienal de Desarrollo en las Provincias de Azuay, Cañar y Morona Santiago. [Informe inédito del CREA para el Banco Interamericano de Desarrollo]. Cuenca, Ecuador.

Disponible en: Archivo del Centro de Reconversión económica del Azuay, Cañar y Morona Santiago, Sede Senplades Zona 6-Austro, Cuenca.

Meót, H.J.A. (1975a). El Concepto de Región. Documentos Complementarios. [Notas técnicas inéditas]. Santiago de Chile: CEPAL.

Disponible en: <http://hdl.handle.net/11362/33679>

Meót, H.J.A. (1975b). Diagnostico Espacial del Ecuador: Método de diseño de las zonas de polarización de los centros poblados. [Notas técnicas inéditas]. Santiago de Chile: CEPAL.

Disponible en: <http://hdl.handle.net/11362/35022>

Moncada S., J. (1974). La evolución de la planificación en el Ecuador. Nueva Sociedad, 13, $27-45$. 
Moncayo J., E. (2013). La Evolución de las Políticas Regionales en los Países Andinos: Una visión comparativa. Documentos y Aportes en Administración Pública y Gestión Estatal, 21 (13), 7-39. <http://www.uacm.kirj.redalyc.redalyc.org/articulo. oa?id=337530225001>

Ojeda S., L. (2000). La descentralización en el Ecuador. Avatares de un proceso inconcluso. Quito: Abya-Yala.

Pauta C., F. (2013). Ordenación Territorial y Urbanística: Un camino para su aplicación en el Ecuador. Cuenca: Monsalve Moreno.

Peñaherrera P., B. (1986). Apuntes para la historia de la planificación en el Ecuador. En Malo G., C. (Ed.). Ecuador Contemporáneo (pp. 75-94). México: Unam, 1986.

Planeación Regional para propender el Desarrollo Armónico e Integral del País. (1968, 12 de abril). El Comercio, p. 17.

Regalado, J. F. (2011). Aproximación a las políticas de planificación y desarrollo en Ecuador y sus fundamentos sociales desde Azuay (1930-1960). Pucara, 24, 135154.

República del Ecuador. (2017). Plan Nacional para el Buen Vivir 2017-2021. Quito: SENPLADES. $159 \mathrm{p}$.

Rusque, J. A. (1976). Ecuador. Bases para la implementación de un sistema de participación de la población en el proceso de Planificación Regional del Ecuador. [Proyecto de Planificación Regional/Nacional ECU/74/005]. Quito: JUNAPLA y ONU.

Valarezo, G. R., Báez Rivera, S. y Ospina Peralta, P. (2004). Una breve historia del espacio ecuatoriano. Quito: Instituto de Estudios Ecuatorianos-IEE.

Washima Ch., O. (1981). Alcances y limitaciones de la Planificación Regional en el Ecuador: Evaluación de una experiencia. En CREA (Ed.), Memoria: Primer Encuentro Nacional de Planificación Regional y Desarrollo (pp. 81-109). Cuenca: CREA-Dirección de Planificación.

Disponible en: Archivo del Centro de Reconversión económica del Azuay, Cañar y Morona Santiago, Sede Senplades Zona 6-Austro, Cuenca. 